IJMMS 29:10 (2002) 613-624

PII. S0161171202011870

http://ijmms.hindawi.com

(c) Hindawi Publishing Corp.

\title{
SZEGŐ PROJECTIONS FOR HARDY SPACES OF MONOGENIC FUNCTIONS AND APPLICATIONS
}

\author{
SWANHILD BERNSTEIN and LOREDANA LANZANI
}

Received 6 February 2001 and in revised form 12 June 2001

\begin{abstract}
We introduce Szegő projections for Hardy spaces of monogenic functions defined on a bounded domain $\Omega$ in $\mathbb{R}^{n}$. We use such projections to obtain explicit orthogonal decompositions for $L^{2}(b \Omega)$. As an application, we obtain an explicit representation of the solution of the Dirichlet problem for balls and half spaces with $L^{2}$. Clifford algebra-valued, boundary datum.
\end{abstract}

2000 Mathematics Subject Classification: 30G35, 30C40, 31B10, 31A10, 31A25.

1. Introduction. This note is aimed to a mixed audience of complex analysts and Clifford analysts. Complex analysts should be interested in the idea that certain features of complex function theory in the plane which are lost in higher dimensions can be recovered by embedding $\mathbb{C}^{n}$ into a Clifford algebra of suitable dimension. We also hope to attract more Clifford analysts to the study of Szegó projections onto Hardy spaces of monogenic functions; we expect that this subject will lead to interesting applications in the areas of partial differential equations and boundary value problems.

The origins of the questions raised in this note go back to earlier work of Bell on the study of the Szegő projection for a smooth, bounded, simply connected domain in the complex plane. More precisely, in [3], Bell has produced a new method yielding the explicit representation plus existence and regularity for the solution of the classical Dirichlet problem (see Section 2).

The formulas obtained by Bell are very elegant and highly explicit but have the shortcoming of being confined to the two-dimensional setting. This is because a harmonic function of several complex variables needs not be the real part of a holomorphic function (not even locally), and very simple examples can be exhibited to this extent (see [23]).

The question then arises whether this obstacle may be circumvented by embedding $\mathbb{C}^{n}$ into some larger environment, one where the solution of the Dirichlet problem can be represented in terms of a suitable analog of the Szegó projection. Clifford algebras appear to be a natural candidate for doing so, as under many respects their structure resembles the complex plane. For example, it is true that in certain domains in $\mathbb{R}^{n}$ a (scalar-valued) harmonic function can be expressed as the scalar part of a two-sided monogenic function taking values in $C \ell_{(n-1)}$ (see $\left.[11,19]\right)$. However, the noncommutative nature of Clifford algebras raises new obstacles, in particular, the 
striking fact that monogenic functions are no longer an algebra, as the pointwise multiplication of two monogenic functions needs not be monogenic. Moreover, higherdimensional Clifford algebras contain zero divisors, and therefore the problem of studying the invertibility of a $C \ell_{n}$-valued function becomes far more complex than looking for its zeroes.

Our main concern here is to show that Szegó projections onto Hardy spaces of left and right monogenic functions may be effectively used to give orthogonal decompositions for the space $L^{2}(b \Omega)$, where $\Omega \subset \mathbb{R}^{n}$ is a domain with reasonably smooth (i.e., Lipschitz) boundary. Such decompositions extend well-known results for the plane proved by Schiffer in the fifties (see [25]), but in our context a new interplay of left and right operators appears which, in the commutative setting is, of course, unheard of.

As an application, we obtain a new representation of the solution of the $L^{2}$-Dirichlet problem on a ball or a half space in $\mathbb{R}^{n}$ where the boundary datum takes values in $C \ell_{(n-1)}\left(\mathbb{R}\right.$ or $\mathbb{C}$ can, of course, be embedded in $\left.C \ell_{(n-1)}\right)$. The obstacles that one meets when trying to extend this result to an arbitrary domain in $\mathbb{R}^{n}$ (even under the assumption that the boundary be $C^{\infty}$-smooth) lead to several open questions, here we just mention the problem of characterizing the domains for which the Szegö kernel is a (nonvanishing) invertible function with monogenic inverse.

The structure of this note is as follows. In Section 2, we briefly recall the results of Bell which motivated our work. In Section 3, we review the main properties of Clifford algebras that are of interest here. In Section 4, we present two orthogonal decompositions for the space of square-integrable, $C \ell_{n}$-valued functions defined on the boundary of a (Lipschitz) domain in $\mathbb{R}^{n+1}$. Finally, in Section 5 , we show how to use such decompositions to solve the Dirichlet problem for a ball or a half space in $\mathbb{R}^{n+1}$.

2. Bell's theorems for the plane. All the facts and results that are about to be stated in this section are taken from [3] (see also [1, 2]). Let $\Omega \subset \mathbb{C}$ denote a bounded, simply connected domain with $C^{\infty}$-smooth boundary. We define the Hardy space $H^{2}(b \Omega)$ as the (boundary values of the) $L^{2}(b \Omega)$-closure of the space of functions which are analytic in $\Omega$ and of class $C^{\infty}$ up to the boundary. The Hardy space is thus a closed subspace of a Hilbert space and, as such, it has an orthogonal projection $P: L^{2}(b \Omega) \rightarrow H^{2}(b \Omega)$ which is known as the Szegó projection. Such projection has a singular integral representation in terms of the Szegó kernel function $S_{a}(z):=S(z, a)$, $z \in \bar{\Omega}, a \in \Omega$. The orthogonal complement of the Szegó projection also has a singular integral representation whose kernel is known as the Garabedian kernel, $L_{a}$. The properties of $S_{a}$ and $L_{a}$ are strictly connected to the smoothness of the domain. In particular, if the domain is of class $C^{\infty}, S_{a}$ is an analytic function of class $C^{\infty}$ up to the boundary, and $S_{a}(z) \neq 0$ for any $z \in \bar{\Omega}$. The Garabedian kernel $L_{a}$ is also nevervanishing in $\bar{\Omega}$; it is analytic in $\Omega \backslash\{a\}$ and its singularity at $\{a\}$ is precisely $1 /(z-a)$. Moreover, $L_{a}$ is of class $C^{\infty}$ in $\bar{\Omega} \backslash\{a\}$.

The Szegó projection and the Szegó and Garabedian kernels may be used to solve the classical Dirichlet problem.

THEOREM 2.1 (see [3]). Let $\Omega \subset \mathbb{C}$ denote a bounded, simply connected domain with $C^{\infty}$-smooth boundary. Let $a \in \Omega$ be a point fixed arbitrarily and let $\varphi \in C^{\infty}(b \Omega)$ be 
given. Then, the classical Dirichlet problem

$$
\begin{gathered}
\Delta u(z)=0, \quad z \in \Omega, \\
u(z)=\varphi(z), \quad z \in b \Omega
\end{gathered}
$$

has the unique solution

$$
u=h+\bar{H},
$$

where $h$ and $H$ are two analytic functions of class $C^{\infty}$ up to the boundary of $\Omega$, which can be explicitly represented as follows:

$$
h=\frac{P\left(S_{a} \varphi\right)}{S_{a}}, \quad H=\frac{P\left(L_{a} \bar{\varphi}\right)}{L_{a}} .
$$

3. Clifford algebras: background information. We briefly review some basic definitions and properties of quaternionic function theory. A more exhaustive introduction can be found in the excellent books [5, 9] where the reader can find a study of the function theory corresponding to the Dirac operator (and related special functions), as well as a treatment of residual theory. For a point of view closer to harmonic analysis we refer to [10]. A summary of developments in Clifford and quaternionic analysis and its relations to physics (plus some numerical analysis) is contained in [12]. We conclude this list by mentioning that both $[11,15]$ emphasize applications to problems in physics, the latter in greater detail.

Let $\mathbb{R}^{n}$ denote the Euclidean space. The real Clifford Algebra associated with $\mathbb{R}^{n}$, denoted $C \ell_{n}$, is defined as the minimal enlargement of $\mathbb{R}^{n}$ to a unitary algebra not generated by any proper subspace of $\mathbb{R}^{n}$, with the property that $x^{2}=-|x|^{2}$ for any $x \in \mathbb{R}^{n}$. This implies that

$$
e_{j} e_{k}+e_{k} e_{j}=-2 \delta_{j k}, \quad j, k \geq 1,
$$

where $\left\{e_{j}\right\}_{j=1}^{n}$ denote the generating elements of $C \ell_{n}$, which are usually identified with the standard orthonormal basis in $\mathbb{R}^{n}$.

If we let $e_{0}=1$ denote the unit element of the algebra, it is then clear that any element $a \in C \ell_{n}$ can be uniquely represented as

$$
a=\sum_{l=0}^{n} \sum_{|I|=l} a_{I} e_{I}, \quad a_{I} \in \mathbb{R},
$$

where $e_{I}=e_{i_{1}} e_{i_{2}} \cdots e_{i_{l}}, 1 \leq i_{1}<i_{2}<\cdots i_{l} \leq n, I=\left(i_{1}, i_{2}, \ldots, i_{n}\right)$. This means that the algebra $C \ell_{n}$ is a $2^{n}$-dimensional vector space and a basis is given by all possible (ordered) products of the generating elements.

The Clifford conjugation on $C \ell_{n}$ is defined as the unique (real-) linear involution on $C \ell_{n}$ with $\overline{e_{I}} e_{I}=e_{I} \overline{e_{I}}=1$, for all $I$. Thus

$$
\bar{a}=\sum_{l=0}^{n} \sum_{|I|=l} a_{I} \overline{e_{I}}, \quad \overline{e_{I}}=(-1)^{(l(l+1)) / 2} e_{I},|I|=l .
$$

We define the scalar part of $a$, denoted Sc $(a)$, by

$$
\operatorname{Sc}(a)=a_{0} e_{0} .
$$


It is customary to view $\mathbb{R}^{n+1}$ as embedded into $C \ell_{n}$. Then, for $x \in \mathbb{R}^{n+1}$, a Cliffordvalued function $f(x)$ is defined as

$$
f(x)=\sum_{l=0}^{n} \sum_{|I|=l} f_{I}(x) e_{I},
$$

where $f_{I}$ are given scalar-valued functions, that is, $f_{I}: \Omega \rightarrow \mathbb{R}$, where $\Omega$ denotes an open subset in $\mathbb{R}^{n+1}$. Properties such as continuity, differentiability, and so forth which are ascribed to $f(x)$ have to be possessed by all components $f_{I}(x)$.

Next, we introduce the Dirac operator

$$
D:=\sum_{j=0}^{n} e_{j} \frac{\partial}{\partial x_{j}} .
$$

A (Clifford algebra-valued) function $f$ is called left-monogenic in a domain $\Omega \subset \mathbb{R}^{n+1}$ if and only if

$$
D f=\sum_{j=0}^{n} \sum_{l=0}^{n} \sum_{|I|=l} \frac{\partial f_{I}}{\partial x_{j}}(x) e_{j} e_{I}=0, \quad x \in \Omega,
$$

and right-monogenic if and only if

$$
f D=\sum_{j=0}^{n} \sum_{l=0}^{n} \sum_{|I|=l} \frac{\partial f_{I}}{\partial x_{j}}(x) e_{I} e_{j}=0, \quad x \in \Omega .
$$

Due to the noncommutative structure of $C \ell_{n}(n \geq 2)$, a left-monogenic function need not be right monogenic and vice versa. For example, in $C \ell_{2}$, it can be easily verified that the function $w\left(x_{0}, x_{1}, x_{2}\right):=x_{0} e_{0}+\left(x_{0}+x_{2}\right) e_{2}+x_{1} e_{12}$ is left but not right monogenic.

Functions that are both left and right monogenic are called two-sided monogenic. A classical example of an element in this class is given by the Cauchy-kernel in $\mathbb{R}^{n+1}$, namely

$$
e(x)=\frac{1}{\sigma_{n}} \frac{\bar{x}}{|x|^{n+1}}, \quad x \neq 0 .
$$

Here, $\sigma_{n}$ denotes the surface measure of the unit sphere in $\mathbb{R}^{n+1}$. The Cauchy kernel is a fundamental solution of $D$ and it may be used to define several integral operators (see Section 4).

For a $C \ell_{n}$-valued function $u(x)=\sum_{l=0}^{n} \sum_{|I|=l} u_{I}(x) e_{I}$, the Laplacian of $u$, denoted $\Delta u$, is the $C \ell_{n}$-valued function

$$
\Delta u(x):=\sum_{l=0}^{n} \sum_{|I|=l} \Delta u_{I}(x) e_{I}, \quad \Delta u_{I}(x)=\sum_{j=1}^{n} \frac{\partial^{2} u_{I}}{\partial x_{j}^{2}}(x) .
$$

Thus, $u$ is harmonic if and only if all its components so are.

The Dirac operator and the Laplacian are directly connected to one another via the formula

$$
\bar{D} D=D \bar{D}=\Delta
$$

which shows that monogenic functions are harmonic. 
In what follows, we need to be able to connect the behavior of a function inside the domain with the values attained on the boundary. This can be achieved via the Stokes' formula (see [9])

$$
\int_{b \Omega} u(x) n(x) v(x) d \sigma(x)=\iint_{\Omega}(u D)(x) v(x) d x+\iint_{\Omega} u(x)(D v)(x) d x,
$$

where $d \sigma$ denotes surface measure for $b \Omega, d x$ denotes volume measure for $\Omega$, and $n$ denotes the outward normal unit vector.

The Clifford algebra $C \ell_{n}$ and the Dirac operator $D$ can be interpreted as higherdimensional analogs of the complex numbers and the $\bar{\partial}$-operator, respectively. In this sense monogenic functions are the analog of analytic functions in the complex plane. Indeed, in the case $n=1$ the algebra $C \ell_{1}$ coincides with the complex numbers via the obvious identifications $e_{0}:=1$ and $e_{1}:=i$.

However, in higher dimensions the noncommutative structure of the algebras induces fundamental differences with the complex case; in particular, monogenic functions are no longer closed under pointwise Clifford multiplication, and it is very easy to produce examples to this end: the two functions $u\left(x_{0}, x_{1}, x_{2}\right):=x_{1} e_{2}+x_{2} e_{1}$; $v\left(x_{0}, x_{1}, x_{2}\right):=x_{1} e_{1}-x_{2} e_{2}$ are two-sided monogenic but their pointwise multiplication is neither left nor right monogenic. The function $v$ in the example above may also be used to show that neither the square nor the inverse of an (invertible) monogenic function need be monogenic.

Another feature that makes a higher-dimensional Clifford algebra into a quite different environment from the complex space is the existence of zero divisors $(n \geq 3)$; for instance, for the two nonzero elements $w_{1}:=1+e_{1} e_{2} e_{3}, w_{2}:=1-e_{1} e_{2} e_{3} \in C \ell_{3}$ we have $w_{1} w_{2}=0$, as is easily verified. The problem of deciding whether a $C \ell_{n}$-valued function has a multiplicative inverse is therefore more involved than just checking that the function is nonvanishing. Nonetheless, if $u$ is a paravector in $C \ell_{n}$, that is, $u=\sum_{j=0}^{n} u_{j} e_{j}$, then $u$ is invertible if and only if

$$
u \bar{u}=\bar{u} u \neq 0,
$$

and the equality $u \bar{u}=\operatorname{Sc}(u \bar{u})=\operatorname{Sc}(\bar{u} u)=\sum_{j=0}^{n} u_{j}^{2}$ may be used to show that the inverse element is given by

$$
u^{-1}=\frac{\bar{u}}{\bar{u} u} .
$$

In particular, we have that the Cauchy kernel $e(x), x \neq 0$, is an invertible paravectorvalued function.

4. Orthogonal decompositions for spaces of $C \ell_{n}$-valued functions. Let $\Omega \subset \mathbb{R}^{n+1}$ denote an open set with Lipschitz boundary. The space of $C \ell_{n}$-valued, squareintegrable functions on $b \Omega$, denoted $L^{2}(b \Omega)$, is defined as follows:

$$
L^{2}(b \Omega)=\left\{\left.f\left|f: b \Omega \rightarrow C \ell_{n}, \int_{b \Omega}\right| f(x)\right|^{2} d \sigma(x)<+\infty\right\},
$$


where $f(x)=\sum_{l=0}^{n} \sum_{|I|=l} f_{I}(x) e_{I},|f(x)|^{2}=\operatorname{Sc}(f(x) \overline{f(x)})=\sum_{l=0}^{n} \sum_{|I|=l}\left|f_{I}(x)\right|^{2}$. The space $L^{2}(b \Omega)$ is endowed with an inner product

$$
\langle u, v\rangle:=\mathrm{Sc}\left(\int_{b \Omega} \bar{u}(x) v(x) d \sigma(x)\right)=\mathrm{Sc}\left(\int_{b \Omega} u(x) \bar{v}(x) d \sigma(x)\right) .
$$

Two important subspaces of $L^{2}(b \Omega)$ are the so-called left and right Hardy spaces of monogenic functions, namely

$$
\begin{aligned}
& H_{l}^{2}(b \Omega):=\left\{f^{+} \mid f \text { left monogenic in } \Omega, f^{*} \in L^{2}(b \Omega)\right\}, \\
& H_{r}^{2}(b \Omega):=\left\{g^{+} \mid g \text { right monogenic in } \Omega, g^{*} \in L^{2}(b \Omega)\right\} .
\end{aligned}
$$

Here, $h^{+}$and $h^{*}$ denote, respectively, the nontangential limit and the nontangential maximal function of $h$ (see [13]). It is well known that both $H_{l}^{2}(b \Omega)$ and $H_{r}^{2}(b \Omega)$ are closed subspaces of $L^{2}(b \Omega)$ (see [16]). A complete characterization of these spaces may be found in [20].

Next, we introduce a few projection operators of $L^{2}(b \Omega)$ onto the Hardy spaces which will be of central interest to us. In what follows, we let the symbol $e(x-y)$ denote the kernel given in (3.9). Any function denoted by either $u$ or $v$ is assumed to be in $L^{2}(b \Omega)$.

We begin by recalling the classical left and right Cauchy integral operators

$$
\begin{aligned}
& C_{l} v(x):=\int_{b \Omega} e(x-y) n(y) v(y) d \sigma(y), \\
& C_{r} v(x):=\int_{b \Omega} v(y) n(y) e(x-y) d \sigma(y),
\end{aligned}
$$

where $x \in \Omega$.

Left Cauchy integrals are left monogenic in $\Omega$, and right Cauchy integrals are right monogenic. Two versions of the Cauchy integral formula, one for right monogenic functions and another for left monogenic functions are known to be valid in this setting, namely (see [20])

$$
\begin{aligned}
& f(x)=\left(C_{l} f\right)(x), \quad x \in \Omega, f \in C(\bar{\Omega}), D f=0, \\
& g(x)=\left(C_{r} g\right)(x), \quad x \in \Omega, g \in C(\bar{\Omega}), g D=0 .
\end{aligned}
$$

The principal values of the Cauchy integrals give rise to two boundary operators, denoted $K_{l}$ and $K_{r}$, respectively, which are closely related to the Hilbert transform

$$
\begin{aligned}
& K_{l} v(x):=2 \text { p.v. } \int_{b \Omega} e(x-y) n(y) v(y) d \sigma(y), \quad \text { a.e. } x \in b \Omega, \\
& K_{r} v(x):=2 \text { p.v. } \int_{b \Omega} v(y) n(y) e(x-y) d \sigma(y), \quad \text { a.e. } x \in b \Omega,
\end{aligned}
$$

where p.v. denotes principal value.

It is a classical result that if the boundary of $\Omega$ is a smooth surface then both $K_{l}$ and $K_{r}$ are well-defined, bounded operators on $L^{2}(b \Omega)$ (see $[5,9,10,11,12,20]$ ). In fact, in the early eighties, Coifman, McIntosh, and Meyer were able to prove that $K_{l}$ 
and $K_{r}$ are well defined and bounded even when the smoothness of $b \Omega$ is reduced to Lipschitz (see [8] and, for Clifford algebras, [17, 18, 20]). We define the left and right Cauchy transforms, denoted, respectively, $C_{l}^{+}$and $C_{r}^{+}$, as the two boundary operators given by the nontangential limits of the left and right Cauchy integrals. The classical Plemelj formula can be applied to obtain the following explicit representations for the Cauchy transforms (see [20]):

$$
C_{l}^{+} u(x)=\frac{1}{2}\left[\left(I+K_{l}\right) u\right](x), \quad C_{r}^{+} u(x)=\frac{1}{2}\left[\left(I+K_{r}\right) u\right](x), \quad \text { a.e. } x \in b \Omega,
$$

where $I$ denotes the identity on $L^{2}(b \Omega)$. The $L^{2}(b \Omega)$-boundedness of the operators $K_{l}$ and $K_{r}$ together with the monogenicity of the Cauchy integrals imply at once that $C_{l}^{+}$ and $C_{r}^{+}$are bounded projections acting among the following spaces:

$$
C_{l}^{+}: L^{2}(b \Omega) \rightarrow H_{l}^{2}(b \Omega), \quad C_{r}^{+}: L^{2}(b \Omega) \rightarrow H_{r}^{2}(b \Omega) .
$$

Following [3], we now produce explicit representations for the $L^{2}(b \Omega)$-adjoints of these operators.

LEMMA 4.1. With the notations and assumptions above, for any $v \in L^{2}(b \Omega)$ and for a.e. $x \in b \Omega$, the following representation formulas hold:

$$
\begin{aligned}
& \left(C_{l}^{+}\right)^{*} v(x)=\frac{1}{2}\left[v(x)-\bar{n}(x) \overline{K_{r}(\overline{n v})(x)}\right], \\
& \left(C_{r}^{+}\right)^{*} v(x)=\frac{1}{2}\left[v(x)-\overline{K_{l}(\overline{v n})}(x) \bar{n}(x)\right] .
\end{aligned}
$$

Proof. We show how to prove the formula for $\left(C_{l}^{+}\right)^{*}$, the proof for $\left(C_{r}^{+}\right)^{*}$ being similar. Because of the Plemelj formula (4.7) it is clear that all we need to show is the equality $K_{l}^{*} v(x)=-\bar{n}(x) \overline{K_{r}(\overline{n v})}(x)$. In this regard, we have

$$
\begin{aligned}
& \left\langle K_{l} u, v\right\rangle=\mathrm{Sc}\left(\int_{b \Omega} \overline{K_{l} u}(x) v(x) d \sigma(x)\right) \\
& =\operatorname{Sc}\left(\int_{b \Omega} 2 \text { p.v. }\left(\int_{b \Omega} e(x-y) n(y) u(y) d \sigma(y)\right) v(x) d \sigma(x)\right) \\
& =\mathrm{Sc}\left(\int_{b \Omega} \bar{u}(y) \bar{n}(y) 2 \text { p.v. } \int_{b \Omega} \overline{e(x-y)} \bar{n}(x)(n(x) v(x)) d \sigma(x) d \sigma(y)\right) \\
& =\operatorname{Sc}\left(\int_{b \Omega} \bar{u}(y) \bar{n}(y)\left[2 \overline{\text { p.v. } \int_{b \Omega}(\overline{n(x) v(x)}) n(x) e(x-y) d \sigma(x)}\right] d \sigma(y)\right) \\
& =-\mathrm{Sc}\left(\int_{b \Omega} \bar{u}(y) \bar{n}(y)\left[2 \overline{\text { p.v. } \int_{b \Omega}(\overline{n(x) v(x)}) n(x) e(y-x) d \sigma(x)}\right] d \sigma(y)\right) \\
& =\left\langle u,-\bar{n} \overline{K_{r}(\overline{n v})}\right\rangle \text {. }
\end{aligned}
$$

The proof is concluded. 
Lemma 4.1 may be used to characterize the orthogonal complements of the spaces $H_{l}^{2}(b \Omega)$ and $H_{r}^{2}(b \Omega)$, as follows.

LEMMA 4.2. Let $v \in L^{2}(b \Omega)$. Then $v \in\left(H_{l}^{2}(b \Omega)\right)^{\perp}$ if and only if $v=\overline{H n}$ for some $H \in H_{r}^{2}(b \Omega)$, where $n$ denotes the outward normal unit vector.

Proof. We begin by showing that functions of the form $\overline{H n}$, with $H \in H_{r}^{2}(b \Omega)$ are orthogonal to the space $H_{l}^{2}(b \Omega)$. In fact, for any given $u \in H_{l}^{2}(b \Omega)$ and $H \in H_{r}^{2}(b \Omega)$, Stokes' formula (3.12) readily implies that

$$
\langle\overline{H n}, u\rangle=\mathrm{Sc}\left(\int_{b \Omega} H(x) n(x) u(x) d \sigma(x)\right)=0 .
$$

On the other hand, for $v \in\left(H_{l}^{2}(b \Omega)\right)^{\perp}$ and $u \in L^{2}(b \Omega)$ we have $0=\left\langle C_{l}^{+} u, v\right\rangle=$ $\left\langle u,\left(C_{l}^{+}\right)^{*} v\right\rangle$, so that $\left(C_{l}^{+}\right)^{*} v=0$. By applying Lemma 4.1 it thus follows that

$$
v=\bar{n} \overline{K_{r}(\overline{n v})}=\overline{K_{r}(\overline{n v}) n}=\overline{\left(2 C_{r}^{+}(\overline{n v})-\overline{n v}\right) n}=\overline{\left(2\left(C_{r}^{+}(\overline{n v})\right) n-\bar{v}\right)}
$$

and we obtain $v=\overline{H n}$ with $H:=C_{r}^{+}(\overline{n v})$. The proof is concluded.

The following similar result holds for the orthogonal complement of the right Hardy space.

LEMMA 4.3. Let $v \in L^{2}(b \Omega)$. Then $v \in\left(H_{r}^{2}(b \Omega)\right)^{\perp}$ if and only if, $v=\overline{n h}$ for some $h \in H_{l}^{2}(b \Omega)$, where $n$ denotes the outward normal unit vector.

From the formulas in Lemma 4.1 it is clear that, in general, Cauchy transforms are not selfadjoint projections (see also [14]). Because $H_{l}^{2}(b \Omega)$ and $H_{r}^{2}(b \Omega)$ are closed subspaces of $L^{2}(b \Omega)$ the classical theory of Hilbert spaces grants the existence of orthogonal (i.e., selfadjoint) projections onto such subspaces. We call such projections the left and right Szegó projections for $L^{2}(b \Omega)$, denoted $P_{l}$ and $P_{r}$. In short, we have

$$
\begin{gathered}
P_{l}: L^{2}(b \Omega) \longrightarrow H_{l}^{2}(b \Omega), \quad P_{r}: L^{2}(b \Omega) \longrightarrow H_{r}^{2}(b \Omega), \\
\left\|P_{r}\right\|=1=\left\|P_{l}\right\|, \quad \text { that is, } P_{r}=P_{r}^{*}, P_{l}=P_{l}^{*} .
\end{gathered}
$$

Here, the symbol $\|\cdot\|$ denotes operator norm.

Any function $u \in L^{2}(b \Omega)$ admits orthogonal decompositions in terms of $P_{r}, P_{l}$, and their orthogonal complements. These formulas can be made explicit by applying Lemmas 4.2 and 4.3, as follows.

THEOREM 4.4. With the same notations and assumptions as above, any function $u \in L^{2}(b \Omega)$ has the following unique orthogonal decomposition in $L^{2}(b \Omega)$ :

$$
P_{l} u+\overline{P_{r}(\overline{n u}) n}=u=P_{r} u+\overline{n P_{l}(\overline{u n})} .
$$

Proof. It is enough to show the first equality. We have $u=P_{l} u+\left(P_{l}^{\perp} u\right)=P_{l} u+\overline{H n}$, for some $H \in H_{r}^{2}(b \Omega)$ (see Lemma 4.3). Hence, $\bar{u}=\overline{P_{l} u}+H n$, so that $\bar{u} \bar{n}=\overline{P_{l}} u \bar{n}+H$, that is, $\overline{n u}=\overline{n P_{l} u}+H$, with $H \in H_{r}^{2}(b \Omega)$ and $\overline{n P_{l} u} \in H_{r}^{2}(b \Omega)^{\perp}$. The uniqueness property of orthogonal decompositions may now be applied to obtain $H=P_{r}(\overline{n u})$. The proof is concluded. 
We conclude this section by introducing the so-called Kerzman-Stein equations for $P_{l}$ and $P_{r}$. Let $\mathbb{A}_{l}$ and $\mathbb{A}_{r}$ denote, respectively, the left and right Kerzman-Stein operators, that is,

$$
\mathbb{A}_{l}:=\left(C_{l}^{+}\right)^{*}-C_{l}^{+}=\frac{1}{2}\left(K_{l}^{*}-K_{l}\right), \quad \mathbb{A}_{r}:=\left(C_{r}^{+}\right)^{*}-C_{r}^{+}=\frac{1}{2}\left(K_{r}^{*}-K_{r}\right) .
$$

The two Kerzman-Stein operators are $L^{2}(b \Omega)$-bounded and skew-adjoint (see [14]); in particular, we have (see [16] and, for a related result in a different context, [26]).

LEMMA 4.5. Let $\Omega \subset \mathbb{R}^{n+1}$ denote a bounded domain with Lipschitz boundary. With the same notations as above, $I-\mathbb{A}_{l}$ and $I-\mathbb{A}_{r}$ are invertible on $L^{2}(b \Omega)$ (here, I denotes the identity on $L^{2}(b \Omega)$ ).

Lemma 4.5 is the key ingredient for proving the equations of Kerzman and Stein for $P_{l}$ and $P_{r}$.

THEOREM 4.6. With the same notations and assumptions as above,

$$
P_{l}=C_{l}^{+}\left(I-\mathbb{A}_{l}\right)^{-1}, \quad P_{r}=C_{r}^{+}\left(I-\mathbb{A}_{r}\right)^{-1}
$$

Proof. As usual, it suffices to give a proof for just one of the two equations, say the one for $P_{l}$. Let $v$ denote an arbitrary element of $L^{2}(b \Omega)$. We have

$$
\begin{aligned}
{\left[I-\mathbb{A}_{l}\right] v } & =\left[I-\frac{1}{2}\left(K_{l}^{*}-K_{l}\right)\right] v=\frac{1}{2}\left(v-K_{l}^{*} v\right)+\frac{1}{2}\left(v+K_{l} v\right) \\
& =\frac{1}{2}\left(v+\bar{n} \overline{K_{r}(\overline{n v})}\right)+\frac{1}{2}\left(v+K_{l} v\right)=\bar{n} \frac{1}{2}\left(n v+\overline{K_{r}(\overline{n v})}\right)+C_{l}^{+} v \\
& =\bar{n} \frac{1}{2}\left(\overline{(\overline{n v})+K_{r}(\overline{n v})}\right)+C_{l}^{+} v=\bar{n} \overline{C_{l}^{+}(\overline{n v})}+C_{l}^{+} v=C_{l}^{+} v+\overline{C_{r}^{+}(\overline{n v}) n}
\end{aligned}
$$

and $\overline{C_{r}^{+}(\overline{n v})}$ is orthogonal to $H_{l}^{2}(b \Omega)$ (see Lemma 4.2). The conclusion follows by applying $P_{l}$ to the very first and the very last terms in the sequel of the equalities above.

Just as in the case of the plane, the importance of the equations of Kerzman and Stein rests on the fact that they provide a quite explicit representation for the Szegó projections, which are defined only in an abstract way, in terms of the Cauchy transforms which are, instead, completely explicit operators (see (4.6) and (4.7)). But this is not all, as even deeper applications may be obtained by regarding the Kerzman-Stein equations as vehicles to deduce new properties of the Szegó projections from known properties of the corresponding Cauchy transforms, and vice versa (see [1, 3]). This well-known subject eludes our current purposes and we will not discuss it in detail. We just wish to mention that in the case when the domain has $C^{\infty}$-smooth boundary, exactly the same arguments as those given in [14] for the case of the plane may be applied to show that both $\mathbb{A}_{l}$ and $\mathbb{A}_{r}$ are smoothing operators. It thus follows that $P_{l}$ (resp., $P_{r}$ ) and $C_{l}^{+}$(resp., $C_{r}^{+}$) have the same type of regularity, that is, they both map the space $C^{\infty}(b \Omega)$ to the space $C^{\infty}(\bar{\Omega})$ (see $[3,20]$ ). 
5. One application. We finally show in this section how to use Theorem 4.4 to obtain a higher-dimensional generalization of Theorem 2.1 in the case of balls or half spaces. We have the following theorem.

THEOREM 5.1. Let $\Omega=\left\{x \in \mathbb{R}^{n+1}|| x-\left.a\right|^{2}<R^{2}\right\}$ and let $\varphi: b \Omega \rightarrow C \ell_{n}, \varphi \in$ $L^{2}(b \Omega)$, be given. Using the notations introduced in the previous sections, the Dirichlet problem

$$
\Delta u=0 \quad \text { in } \Omega, \quad u^{*} \in L^{2}(b \Omega), \quad u^{+}=\varphi \quad \text { on } b \Omega,
$$

has the unique solution

$$
u(x)=\left(P_{l} \varphi\right)(x)+\overline{\left[P_{r}\left(\overline{\left(\frac{x-a}{R}\right) \varphi}\right)\right](x)\left(\frac{x-a}{R}\right)} .
$$

Here, $u^{*}$ and $u^{+}$denote, respectively, the nontangential maximal function and the nontangential limit of $u$ (see [13]).

Proof. To simplify the notations we assume that $\Omega$ is the unit ball centered at the origin, that is, $a=0$ and $R=1$. In such a case the outward normal unit vector $n(x)$ is equal to $x, x \in b \Omega$, and the orthogonal decomposition (4.14) for the boundary datum $\varphi$ yields

$$
\varphi(x)=\left(P_{l} \varphi\right)(x)+\overline{P_{r}(\overline{x \varphi}) x}, \quad \text { a.e. } x \in b \Omega .
$$

At this point we observe that the right-hand side of the equality above is the sum of two functions well defined in the whole of $\Omega$ which are, indeed, harmonic. In fact, we have

$$
\begin{aligned}
\Delta\left(\overline{P_{r}(\overline{x \varphi}) x}\right) & =\Delta\left(\bar{x} \overline{P_{r}(\overline{x \varphi})}\right) \\
& =(\Delta(\bar{x})) \overline{P_{r}(\overline{x \varphi})}+\bar{x}\left(\Delta\left(\overline{P_{r}(\overline{x \varphi})}\right)\right)+2 \sum_{j=0}^{n}\left(\frac{\partial}{\partial x_{j}} \bar{x}\right)\left(\frac{\partial}{\partial x_{j}} \overline{P_{r}(\overline{x \varphi})}\right) \\
& =2 \sum_{j=0}^{n} \bar{e}_{j}\left(\frac{\partial}{\partial x_{j}} \overline{P_{r}(\overline{x \varphi})}\right)=2 \bar{D}\left(\overline{P_{r}(\overline{x \varphi})}\right)=2 \overline{P_{r}(\overline{x \varphi}) D}=0 .
\end{aligned}
$$

The square-integrability of the nontangential maximal function of the harmonic function obtained by adding $P_{l} \varphi$ and the conjugate of $P_{r}(\overline{x \varphi}) x$ is a consequence of the Kerzman-Stein equations (4.16) and Lemma 4.5 (it is well known that the nontangential maximal function of a Cauchy transform is square integrable, see $[8,16])$. Finally, we observe that the condition on the nontangential limit in (5.1) follows at once from the very definitions of Hardy spaces and of Szegó projections. The proof is concluded.

The case when $\Omega$ is a half space is even easier to treat. For example, for $\Omega=\mathbb{R}_{+}^{n+1}:=$ $\left\{x_{0}>0\right\}$, the same arguments as in Theorem 5.1 yield

$$
u=P_{l} \varphi+\overline{P_{r}(\bar{\varphi})}:=h+\bar{H},
$$

and a formula for arbitrary half spaces can also be derived in a similar fashion. We observe that the two functions $h$ and $H$ in the decomposition above are, respectively, left 
and right monogenic. It is not clear whether the second term in the decomposition (5.2) for a ball is indeed (the conjugate of) a right monogenic function (the classical Leibniz rule does not extend to monogenic functions). Even though balls and half spaces are known to be conformally equivalent, we do not yet know whether the pointwise multiplication of a Szegó projection by the outward normal unit vector satisfies some type of conformal invariance (see $[7,22]$ and, for the conformal invariance of Cauchy integrals on manifold, [6, 24]). Indeed, we believe this would be an interesting problem to be studied.

To treat the case of an arbitrary bounded (smooth) domain in $\mathbb{R}^{n+1}$, we need to overcome the obstacle of not knowing the explicit form of the outward normal unit vector. This can be achieved, for example, by looking at the Szegó and Garabedian kernels associated to the domain. Using the same notations as in Section 2, it is very easy to show that, also for the case of $C \ell_{n}$-valued functions, we have

$$
L_{a}=\overline{S_{a}} \bar{n} \quad \text { a.e. on } b \Omega \text {. }
$$

A formal orthogonal decomposition for the boundary datum $\varphi$ would involve the pointwise multiplication by the inverses of the kernels, namely

$$
\varphi=S_{a}^{-1} P_{l}\left(S_{a} \varphi\right)+\overline{P_{r}\left(\bar{\varphi} L_{a}\right)\left(L_{a}\right)^{-1}} .
$$

In order to make formula (5.7) meaningful, we need therefore to study the highly nontrivial problem of characterizing those domains for which the Szegő and Garabedian kernels are nonvanishing and invertible (see $[4,21]$ and related comments in the introduction). Moreover, in order to apply formula (5.7) to the solution of the Dirichlet problem, a further question needs to be settled, namely characterizing those domains for which the two functions in the right-hand side of (5.7) are harmonic. This might involve the study of the monogenicity of the inverses of the Szegó and the Garabedian kernels (see again the comments in the introduction).

ACKNOWLEDGMents. The authors wish to thank J. Ryan for helpful conversations. This paper was written when S. Bernstein was visiting the University of Arkansas at Fayetteville, supported by a Feodor Lynen fellowship of the Alexander von Humboldt Foundation, Bonn, Germany. L. Lanzani was partially supported by an ASTA grant no. 98-B-39.

\section{REFERENCES}

[1] S. Bell, The Szegó projection and the classical objects of potential theory in the plane, Duke Math. J. 64 (1991), no. 1, 1-26.

[2] S. R. Bell, Solving the Dirichlet problem in the plane by means of the Cauchy integral, Indiana Univ. Math. J. 39 (1990), no. 4, 1355-1371.

[3] _ The Cauchy Transform, Potential Theory, and Conformal Mapping, Studies in Advanced Mathematics, CRC Press, Florida, 1992.

[4] H. P. Boas, S. Fu, and E. J. Straube, The Bergman kernel function: explicit formulas and zeroes, Proc. Amer. Math. Soc. 127 (1999), no. 3, 805-811.

[5] F. Brackx, R. Delanghe, and F. Sommen, Clifford Analysis, Research Notes in Mathematics, vol. 76, Pitman, Massachusetts, 1982.

[6] D. Calderbank, Clifford analysis for Dirac operators on manifolds with boundary, preprint, 1997. 
[7] J. Cnops, Reproducing kernels and conformal mappings in $\mathbf{R}^{n}$, J. Math. Anal. Appl. 220 (1998), no. 2, 571-584.

[8] R. R. Coifman, A. McIntosh, and Y. Meyer, L'intégrale de Cauchy définit un opérateur borné sur $L^{2}$ pour les courbes lipschitziennes, Ann. of Math. (2) 116 (1982), no. 2, 361-387 (French).

[9] R. Delanghe, F. Sommen, and V. Souček, Clifford Algebra and Spinor-Valued Functions. A Function Theory for the Dirac Operator, Mathematics and Its Applications, vol. 53, Kluwer Academic Publishers Group, Dordrecht, 1992.

[10] J. E. Gilbert and M. A. M. Murray, Clifford Algebras and Dirac Operators in Harmonic Analysis, Cambridge Studies in Advanced Mathematics, vol. 26, Cambridge University Press, Cambridge, 1991.

[11] K. Gürlebeck and W. Sprössig, Quaternionic Analysis and Elliptic Boundary Value Problems, International Series of Numerical Mathematics, vol. 89, Birkhäuser Verlag, Basel, 1990.

[12] _ Quaternionic and Clifford Calculus for Engineers and Physicists, Wiley \& Sons, New York, 1997.

[13] C. E. Kenig, Harmonic Analysis Techniques for Second Order Elliptic Boundary Value Problems, Regional Conference Series in Mathematics, vol. 83, American Mathematical Society, Rhode Island, 1994.

[14] N. Kerzman and E. M. Stein, The Cauchy kernel, the Szegö kernel, and the Riemann mapping function, Math. Ann. 236 (1978), no. 1, 85-93.

[15] V. V. Kravchenko and M. V. Shapiro, Integral Representations for Spatial Models of Mathematical Physics, Longman, Harlow, 1996.

[16] L. Lanzani, Cauchy transform and Hardy spaces for rough planar domains, Analysis, Geometry, Number Theory: The Mathematics of Leon Ehrenpreis (Philadelphia, PA, 1998), American Mathematical Society, Rhode Island, 2000, pp. 409-428.

[17] C. Li, A. McIntosh, and T. Qian, Clifford algebras, Fourier transforms and singular convolution operators on Lipschitz surfaces, Rev. Mat. Iberoamericana 10 (1994), no. 3, 665-721.

[18] C. Li, A. McIntosh, and S. Semmes, Convolution singular integrals on Lipschitz surfaces, J. Amer. Math. Soc. 5 (1992), no. 3, 455-481.

[19] M. Mitrea, personal communication.

[20] Clifford Wavelets, Singular Integrals, and Hardy Spaces, Lecture Notes in Mathematics, vol. 1575, Springer-Verlag, Berlin, 1994.

[21] A. Nagel, J.-P. Rosay, E. M. Stein, and S. Wainger, Estimates for the Bergman and Szegó kernels in $\mathbf{C}^{2}$, Ann. of Math. (2) 129 (1989), no. 1, 113-149.

[22] T. Qian and J. Ryan, Conformal transformations and Hardy spaces arising in Clifford analysis, J. Operator Theory 35 (1996), no. 2, 349-372.

[23] R. M. Range, Holomorphic Functions and Integral Representations in Several Complex Variables, Graduate Texts in Mathematics, vol. 108, Springer-Verlag, New York, 1986.

[24] J. Ryan, Dirac operators, conformal transformations and aspects of classical harmonic analysis, J. Lie Theory 8 (1998), no. 1, 67-82.

[25] M. Schiffer, Various types of orthogonalization, Duke Math. J. 17 (1950), 329-366.

[26] S. Semmes, Chord-arc surfaces with small constant. I, Adv. Math. 85 (1991), no. 2, 198223.

SWANHILD BERNSTEIN: INSTITUTE OF MATHEMATICS AND PHYSICS, BAUHAUS-UNIVERSITÄT WEIMAR, COUDRAYSTR. 13B, 99421 WEIMAR, GERMANY

E-mail address: bernstei@sfb.uni-weimar.de

LOREDANA LANZANI: DEPARTMENT OF MATHEMATICS, THE UNIVERSITY OF ARKANSAS AT

FAYETTEVILlE, FAYETTEVILLE, AR 72701, USA

E-mail address: 1anzani@comp.uark.edu 


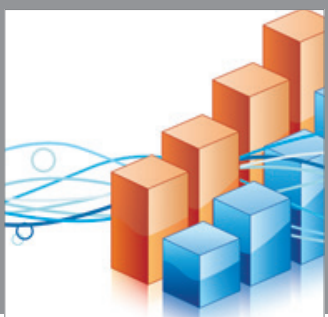

Advances in

Operations Research

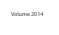

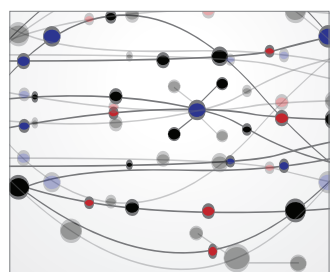

\section{The Scientific} World Journal
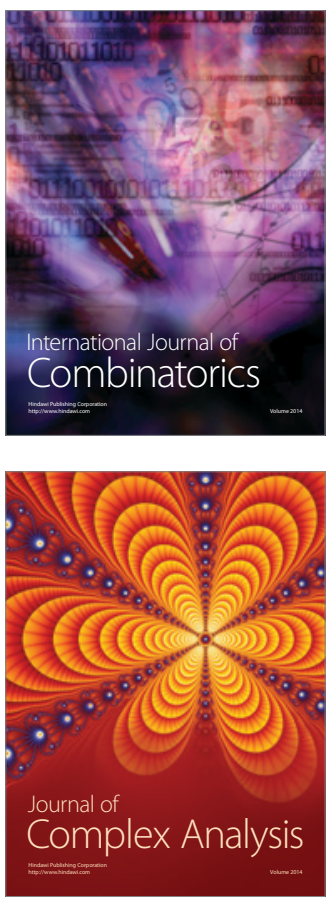

International Journal of

Mathematics and

Mathematical

Sciences
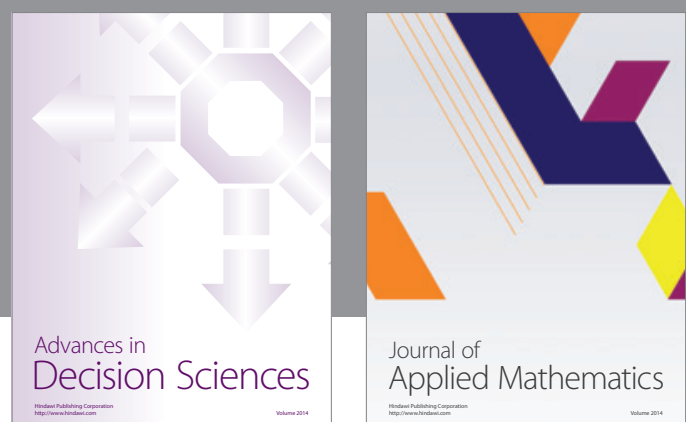

Journal of

Applied Mathematics
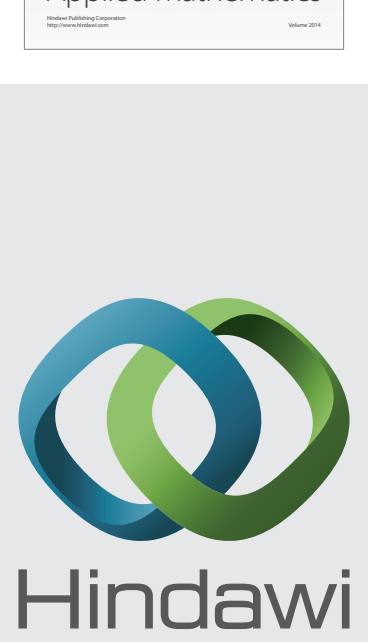

Submit your manuscripts at http://www.hindawi.com
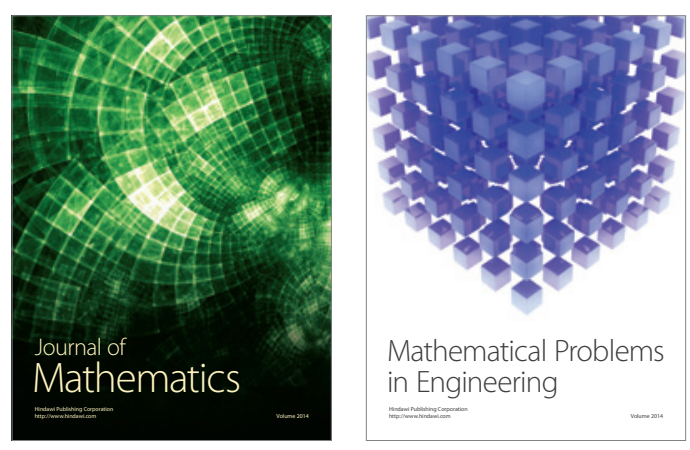

Mathematical Problems in Engineering
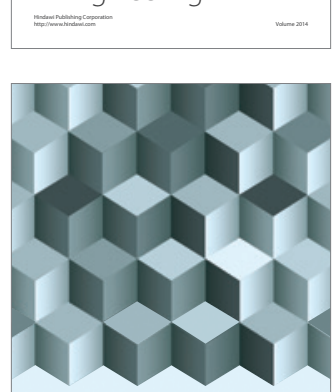

Journal of

Function Spaces
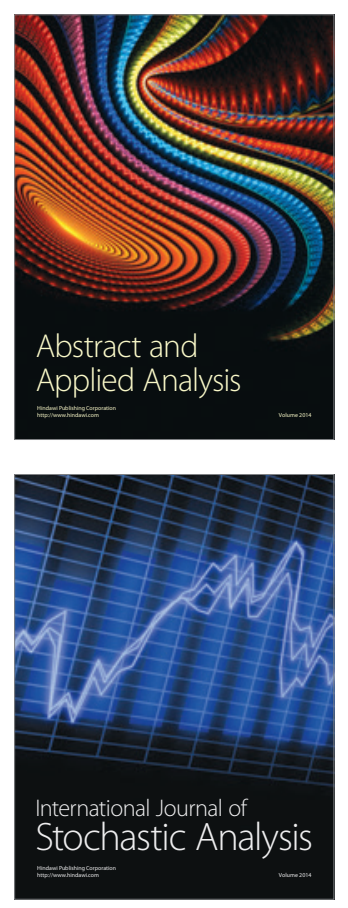

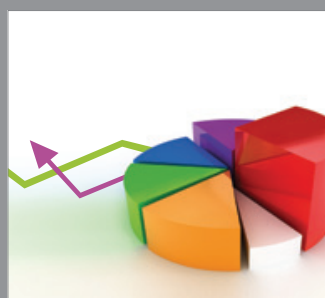

ournal of

Probability and Statistics

Promensencen
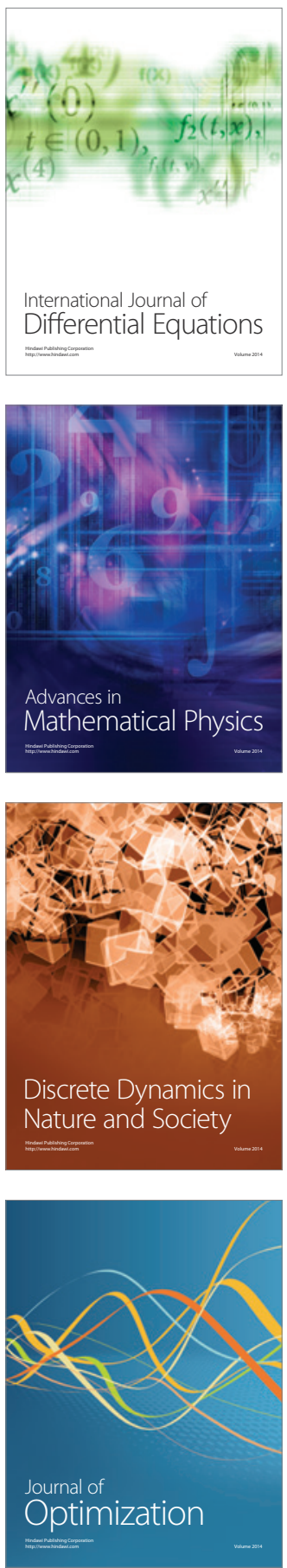